Article

\title{
New Bounds for Topological Indices on Trees through Generalized Methods
}

\author{
Álvaro Martínez-Pérez ${ }^{1}\left([)\right.$ and José M. Rodríguez ${ }^{2, *}$ ([) \\ 1 Departamento de Análisis Económico y Finanzas, Universidad de Castilla-La Mancha, Avda. Real Fábrica \\ de Sedas s/n, 45600 Talavera de la Reina, Spain; alvaro.martinezperez@uclm.es \\ 2 Departamento de Matemáticas, Universidad Carlos III de Madrid, Avenida de la Universidad 30, Leganés, \\ 28911 Madrid, Spain \\ * Correspondence: jomaro@math.uc3m.es; Tel.: +34-925-721-010
}

Received: 3 June 2020; Accepted: 26 June 2020; Published: 2 July 2020

check for updates

\begin{abstract}
Topological indices are useful for predicting the physicochemical behavior of chemical compounds. A main problem in this topic is finding good bounds for the indices, usually when some parameters of the graph are known. The aim of this paper is to use a unified approach in order to obtain several new inequalities for a wide family of topological indices restricted to trees and to characterize the corresponding extremal trees. The main results give upper and lower bounds for a large class of topological indices on trees, fixing or not the maximum degree. This class includes the first variable Zagreb, the Narumi-Katayama, the modified Narumi-Katayama and the Wiener index.

Keywords: first variable zagreb index; Narumi-Katayama index; modified Narumi-Katayama index; Wiener index; topological indices; Schur-convexity; trees
\end{abstract}

MSC: 05C07; 92E10

\section{Introduction}

A topological descriptor is a number computed from a molecular graph representing some information of the corresponding chemical compound. These descriptors are commonly used in mathematical chemistry, and in particular, in the QSPR/QSAR research. A topological index is a topological descriptor that correlates with a physicochemical property of some molecule. Topological indices are useful for predicting the physicochemical behavior of chemical compounds.

The Wiener index of a graph $G$ was defined in [1]. There are many generalizations of this index; see [2-9].

After this original work, many topological indices have been defined. A common strategy to define a topological index is to consider some computation with the end-vertex degrees of edges. This approach has given some particularly useful indices. Possibly the best known of them is the Randic connectivity index (see [10]). There are more than thousand papers and a couple of books dealing with this molecular descriptor (see, e.g., [11-15] and the references therein). Additionally, Gutman et al. defined the first and second Zagreb indices, denoted respectively as $M_{1}$ and $M_{2}$ (see [16]).

The first and the second variable Zagreb indices were introduced in [17] by Miličević and Nikolić as

$$
M_{1}^{\alpha}(G)=\sum_{u \in V(G)} d_{u}^{\alpha}, \quad M_{2}^{\alpha}(G)=\sum_{u v \in E(G)}\left(d_{u} d_{v}\right)^{\alpha},
$$

where $\alpha \in \mathbb{R}, u v$ denotes the edge joining the vertices $u$ and $v$ in the graph $G$, and $d_{u}$ denotes the degree of $u$. There are many papers dealing with these indices; see, e.g., [18-24]. 
Note that $M_{1}^{0}$ is $n, M_{1}^{1}$ is $2 m, M_{1}^{2}$ is the first Zagreb index $M_{1}, M_{1}^{-1}$ is the inverse index ID [25], $M_{1}^{3}$ is the forgotten index $F$, etc.; additionally, $M_{2}^{0}$ is $m, M_{2}^{-1 / 2}$ is the Randić index, $M_{2}^{1}$ is the second Zagreb index $M_{2}, M_{2}^{-1}$ is the modified Zagreb index [26], etc.

Variable molecular descriptors were introduced to characterize heteroatoms in molecules (see $[27,28]$ ). Additionally, they are useful for studying structural differences in the molecule (for example, the role of carbon atoms in alkylcycloalkanes in [29]). In these descriptors, the variables can be fixed along the regression. This way, the error produced estimating some property can be reduced. For example, Gutman and Tosovic compared in [30] the correlation between 20 (vertex-degree-based) topological indices and the standard heat of formation and boiling points of octane isomers. They proved that the second variable Zagreb index $M_{2}^{\alpha}$ with exponent $\alpha=-1$ improved the performance in this case from the Randić index $\left(R=M_{2}^{-1 / 2}\right)$. Ref. [31] shows that the second variable Zagreb index is used in the structure-boiling point modeling of benzenoid hydrocarbons. Several properties of these indices are discussed in several papers (see, e.g., [32-37]).

Among the many uses of topological indices we shall specially mention the medical and pharmacological applications. Moreover, in the 1990s, the field lived an important expansion with many published works. We may cite at least the publications by Erdös, [38,39].

A typical problem in this topic is finding good bounds for the indices, usually when some parameters of the graph (the diameter, the girth, the maximum or minimum degree, etc.) are known. The aim of this paper is to use a unified approach in order to prove new inequalities when the graph is a tree for a wide family of topological indices. Additionally, we try to characterize those trees which are extremal for them. The main results give upper and lower bounds for a wide class of topological indices when the graph is a tree, fixing or not the maximum degree. This class includes the first variable Zagreb, the Narumi-Katayama, the modified Narumi-Katayama and the Wiener index. Our unified approach allows us to obtain quite a few inequalities at once; some were already known, but many are new.

The outline of the paper is as follows. In Section 2 we obtain, by using Schur-convexity, the extremal trees with $n$ vertices for a large family of topological indices (see Theorems 1-4); these results are applied for the first variable Zagreb, the Narumi-Katayama and the modified Narumi-Katayama indices in Theorems 5-8. The Schur-convexity also allows us to find the extremal trees with $n$ vertices and maximum degree $\Delta$ for the previous family of topological indices (see Theorems 9-12); these results are applied for the first variable Zagreb, the Narumi-Katayama and the modified Narumi-Katayama indices in Theorems 13-16. Section 3 contains the results on extremal trees with $n$ vertices for a large class of topological indices, including the Wiener index and many generalizations of this index (see Theorems 17 and 18); Theorems 19 and 20 provide similar results for trees with $n$ vertices and maximum degree $\Delta$.

Herein, $G=(V(G), E(G))$ will denote a non-oriented, finite, connected, simple (i.e., without multiple edges and loops), non-trivial (i.e., $E(G) \neq \varnothing$ ) graph. Notice that asking the graph $G$ to be connected is not a relevant restriction, since any graph representing a molecule is connected. $T$ denotes a tree; i.e., a graph without cycles. Throughout this work, $m$ will denote the cardinality of $E(G)$ and $n$ the cardinality of $V(G)$.

\section{First Variable Zagreb Index}

Given two $n$-tuples $\mathbf{x}=\left(x_{1}, \ldots, x_{n}\right), \mathbf{y}=\left(y_{1}, \ldots, y_{n}\right)$ with $x_{1} \geq x_{2} \geq \cdots \geq x_{n}$ and $y_{1} \geq y_{2} \geq$ $\cdots \geq y_{n}$, then $\mathbf{x}$ majorizes $\mathbf{y}$ (and we write $\mathbf{x} \succ \mathbf{y}$ or $\mathbf{y} \prec \mathbf{x}$ ) if

$$
\sum_{i=1}^{k} x_{i} \geq \sum_{i=1}^{k} y_{i}
$$

for $1 \leq k \leq n-1$ and

$$
\sum_{i=1}^{n} x_{i}=\sum_{i=1}^{n} y_{i}
$$


A function $\Phi: \mathbb{R}^{n} \rightarrow \mathbb{R}$ is said to be Schur-convex if $\Phi(\mathbf{x}) \geq \Phi(\mathbf{y})$ for all $\mathbf{x} \succ \mathbf{y}$. Similarly, $\Phi$ is Schur-concave if $\Phi(\mathbf{x}) \leq \Phi(\mathbf{y})$ for all $\mathbf{x} \succ \mathbf{y}$. We say that $\Phi$ is strictly Schur-convex (respectively, strictly Schur-concave) if $\Phi(\mathbf{x})>\Phi(\mathbf{y})$ (respectively, $\Phi(\mathbf{x})<\Phi(\mathbf{y})$ ) for all $\mathbf{x} \succ \mathbf{y}$ with $\mathbf{x} \neq \mathbf{y}$.

If

$$
\Phi(\mathbf{x})=\sum_{i=1}^{n} f\left(x_{i}\right),
$$

where $f$ is a convex (respectively, concave) function defined on a real interval, then $\Phi$ is Schur-convex (respectively, Schur-concave). If $f$ is strictly convex (respectively, strictly concave), then $\Phi$ is strictly Schur-convex (respectively, strictly Schur-concave).

Thus, by (1) and (2), with $f(x)=x^{\alpha}$,

$$
M_{1}^{\alpha}(G)=\sum_{u \in V(G)} d_{u}^{\alpha}
$$

is strictly Schur-convex if $\alpha \in(-\infty, 0) \cup(1, \infty)$ and strictly Schur-concave if $\alpha \in(0,1)$.

Given $n \geq 2$, let $S_{2 n-2}$ be the set of $n$-tuples $\mathbf{x}=\left(x_{1}, x_{2}, \ldots, x_{n-2}, 1,1\right)$ with $x_{i} \in \mathbb{Z}^{+}$such that $x_{1} \geq x_{2} \geq \cdots \geq x_{n-2} \geq 1$ and $\sum_{i=1}^{n-2} x_{i}=2 n-4$ (i.e., $\sum_{i=1}^{n} x_{i}=2 n-2$ ).

Remark 1. Consider any tree $T$ with $n$ vertices $v_{1}, \ldots, v_{n}$, ordered in such a way that if $\mathbf{x}=\mathbf{x}_{T}=\left(x_{1}, \ldots, x_{n}\right)$ is the $n$-tuple where $x_{i}$ is the degree of the vertex $v_{i}$, then $x_{i} \geq x_{i+1}$ for every $1 \leq i \leq n-1$. By handshaking Lemma, it is urgent to check that $\mathbf{x} \in S_{2 n-2}$.

Lemma 1. If $T$ is a tree with $n \geq 3$ vertices, then

$$
(2, \ldots, 2,1,1) \prec \mathbf{x}_{T} \prec(n-1,1, \ldots, 1) .
$$

Proof. Given a tree $T$, let us consider $\mathbf{x}=\mathbf{x}_{T}=\left(x_{1}, \ldots, x_{n}\right)$. Since $T$ is connected and $n \geq 3$, we have $x_{1} \geq 2$.

Seeking for a contradiction assume that $\sum_{i=1}^{k} x_{i}<2 k$ for some $1 \leq k \leq n-2$. Thus, $x_{k}=1$, and so, $x_{i}=1$ for every $k \leq i \leq n$, and

$$
\sum_{i=1}^{n} x_{i}<2 k+n-k=n+k \leq 2 n-2
$$

leading to a contradiction. Therefore,

$$
\sum_{i=1}^{k} x_{i} \geq 2 k=\sum_{i=1}^{k} 2
$$

for every $1 \leq k \leq n-2$, and so,

$$
(2, \ldots, 2,1,1) \prec \mathbf{x} .
$$

Since

$$
\sum_{i=k+1}^{n} x_{i} \geq \sum_{i=k+1}^{n} 1=n-k
$$

for any $1 \leq k \leq n-1$, we have

$$
\sum_{i=1}^{k} x_{i}=2 n-2-\sum_{i=k+1}^{n} x_{i} \leq n+k-2=n-1+\sum_{i=2}^{k} 1,
$$

where we use the convention $\sum_{i=j}^{k} a_{i}=0$ when $j>k$, as usual. 
Thus,

$$
\mathbf{x} \prec(n-1,1, \ldots, 1) .
$$

Given any function $f:[1, \infty) \rightarrow \mathbb{R}$, let us define the index

$$
I_{f}(G)=\sum_{u \in V(G)} f\left(d_{u}\right)
$$

Besides, if $f$ takes positive values, then we can define the index

$$
I I_{f}(G)=\prod_{u \in V(G)} f\left(d_{u}\right)
$$

Lemma 1 implies the following results.

Theorem 1. If $T$ is a tree with $n \geq 2$ vertices and $f:[1, \infty) \rightarrow \mathbb{R}$ is a convex function, then

$$
(n-2) f(2)+2 f(1) \leq I_{f}(T) \leq f(n-1)+(n-1) f(1) .
$$

Moreover, if $f$ is a strictly convex function, then the lower bound is attained if and only if $T$ is the path graph, and the upper bound is attained if and only if $T$ is the star graph.

Theorem 2. If $T$ is a tree with $n \geq 2$ vertices and $f:[1, \infty) \rightarrow \mathbb{R}$ is a concave function, then

$$
f(n-1)+(n-1) f(1) \leq I_{f}(T) \leq(n-2) f(2)+2 f(1) .
$$

Moreover, if $f$ is a strictly concave function, then the lower bound is attained if and only if $T$ is the star graph, and the upper bound is attained if and only if $T$ is the path graph.

Since the logarithm is a strictly increasing function, a tree is extremal for $I I_{f}(T)$ if and only if it is extremal for

$$
\log I I_{f}(T)=\sum_{u \in V(G)} \log f\left(d_{u}\right) .
$$

Thus, Lemma 1 and (3) imply the following results.

Theorem 3. If $T$ is a tree with $n \geq 2$ vertices and $f:[1, \infty) \rightarrow \mathbb{R}^{+}$is a function such that $\log f$ is convex, then

$$
f(2)^{n-2} f(1)^{2} \leq I I_{f}(T) \leq f(n-1) f(1)^{n-1} .
$$

Moreover, if $\log f$ is a strictly convex function, then the lower bound is attained if and only if $T$ is the path graph, and the upper bound is attained if and only if $\mathrm{T}$ is the star graph.

Theorem 4. If $T$ is a tree with $n \geq 2$ vertices and $f:[1, \infty) \rightarrow \mathbb{R}^{+}$is a function such that $\log f$ is concave, then

$$
f(n-1) f(1)^{n-1} \leq I I_{f}(T) \leq f(2)^{n-2} f(1)^{2} .
$$

Moreover, if $\log f$ is a strictly concave function, then the lower bound is attained if and only if $T$ is the star graph, and the upper bound is attained if and only if $T$ is the path graph.

Since $t^{\alpha}$ is strictly convex if $\alpha \in(-\infty, 0) \cup(1, \infty)$ and strictly concave if $\alpha \in(0,1)$, Theorems 1 and 2 imply, respectively, the following results. 
Theorem 5. If $T$ is a tree with $n \geq 2$ vertices and $\alpha \in(-\infty, 0) \cup(1, \infty)$, then

$$
(n-2) 2^{\alpha}+2 \leq M_{1}^{\alpha}(T) \leq(n-1)^{\alpha}+n-1
$$

Moreover, the lower bound is attained if and only if $T$ is the path graph, and the upper bound is attained if and only if $T$ is the star graph.

The lower bound in Theorem 5 appears in [40].

Theorem 6. If $T$ is a tree with $n \geq 2$ vertices and $\alpha \in(0,1)$, then

$$
(n-1)^{\alpha}+n-1 \leq M_{1}^{\alpha}(T) \leq(n-2) 2^{\alpha}+2
$$

Moreover, the lower bound is attained if and only if $T$ is the star graph, and the upper bound is attained if and only if $T$ is the path graph.

The upper bound in Theorem 6 appears in [40].

Note that the cases $\alpha=0$ and $\alpha=1$ are trivial, since $M_{1}^{0}(G)=|V(G)|$ and $M_{1}^{1}(G)=2|E(G)|$ for every graph $G$.

Theorem 5 has the following consequences.

Corollary 1. If $T$ is a tree with $n \geq 2$ vertices, then the following inequalities hold:

$$
\begin{aligned}
4 n-6 & \leq M_{1}(T) \leq n^{2}-n, \\
8 n-14 & \leq F(T) \leq(n-1)^{3}+n-1, \\
\frac{n}{2}+1 & \leq I D(T) \leq \frac{1}{n-1}+n-1 .
\end{aligned}
$$

Moreover, each lower bound is attained if and only if $T$ is the path graph and each upper bound is attained if and only if $T$ is the star graph.

The inequalities for $M_{1}(T)$ in Corollary 1 appear in [41].

Corollary 2. If $T$ is a tree with $n$ vertices and $\alpha<1$, then

$$
M_{1}^{\alpha}(T)=O(n)
$$

The Narumi-Katayama index was defined in [42] as

$$
N K(G)=\prod_{u \in V(G)} d_{u}
$$

Based on this, it was defined in [43] the modified Narumi-Katayama index

$$
N K^{*}(G)=\prod_{u \in V(G)} d_{u}^{d_{u}}
$$

Since $t \log t$ is a strictly convex function and $\log t$ is a strictly concave function, Theorems 3 and 4 imply, respectively, the following results.

Theorem 7. If $T$ is a tree with $n \geq 2$ vertices, then

$$
2^{2 n-4} \leq N K^{*}(T) \leq(n-1)^{n-1}
$$


Moreover, the lower bound is attained if and only if $\mathrm{T}$ is the path graph, and the upper bound is attained if and only if $T$ is the star graph.

Theorem 8. If $T$ is a tree with $n \geq 2$ vertices, then

$$
n-1 \leq N K(T) \leq 2^{n-2} .
$$

Moreover, the lower bound is attained if and only if $T$ is the star graph, and the upper bound is attained if and only if $T$ is the path graph.

Theorems 7 and 8 were proven in [43,44], respectively, with different arguments.

Given $n \geq 3$ and $2 \leq \Delta \leq n-1$, let $S_{2 n-2}^{\Delta}$ be the set of $n$-tuples $\mathbf{x}=\left(x_{1}, x_{2}, \ldots, x_{n-2}, 1,1\right) \in S_{2 n-2}$ with $x_{1}=\Delta$.

For any $t \in \mathbb{R}$, we denote it as usual by $\lfloor t\rfloor$, the lower integer part of $t$; i.e., the greater integer less than or equal to $t$.

Lemma 2. Let $n \geq 3$ and $2 \leq \Delta \leq n-1$. If $j_{0}=\left\lfloor\frac{n-2}{\Delta-1}\right\rfloor, \mathbf{y}=\left(y_{1}, y_{2}, \ldots, y_{n}\right)$ is such that

- $y_{j}=\Delta$ for every $1 \leq j \leq j_{0}$;

- $y_{j_{0}+1}=2 n-2-j_{0} \Delta-\left(n-j_{0}-1\right)=n-1-j_{0}(\Delta-1)$;

- $y_{j}=1$ for every $j_{0}+1<j \leq n$;

and $\mathbf{z}=\left(z_{1}, z_{2}, \ldots, z_{n}\right)$ is such that

- $\quad z_{1}=\Delta$

- $z_{j}=2$ for every $1<j \leq n-\Delta$;

- $z_{j}=1$ for every $n-\Delta<j \leq n$;

then $\mathbf{y}, \mathbf{z} \in S_{2 n-2}^{\Delta}$ and

$$
\mathbf{z} \prec \mathbf{x} \prec \mathbf{y}
$$

for all $\mathbf{x} \in S_{2 n-2}^{\Delta}$.

Proof. First of all, note that $1 \leq j_{0} \leq n-2$ : Since $\Delta \leq n-1$, it is trivial to check that $j_{0} \geq 1$. Since $j_{0}(\Delta-1) \leq n-2$ and $\Delta \geq 2$, we conclude that $j_{0} \leq n-2$.

Additionally notice that, since $j_{0} \Delta \leq n-2+j_{0}$, we have $2 n-2-j_{0} \Delta-\left(n-j_{0}-1\right) \geq 1$, and since $\left(j_{0}+1\right) \Delta>n-2 \geq n-j_{0}-1$, then $\Delta>n-j_{0} \Delta+j_{0}-1$ and $2 n-2-j_{0} \Delta-\left(n-j_{0}-1\right)<\Delta$. Let us check that $j_{0} \leq n-\Delta$. Since $n \geq \Delta+1$, we have

$$
\begin{aligned}
\Delta(\Delta-1) & \leq n(\Delta-2)+2 \\
n-2 & \leq(n-\Delta)(\Delta-1) \\
j_{0} & \leq \frac{n-2}{\Delta-1} \leq n-\Delta .
\end{aligned}
$$

Thus, $y_{n-1}=1$ if $\Delta \geq 3$. If $\Delta=2$, then $j_{0}=n-2$,

$$
y_{n-1}=y_{j_{0}+1}=n-1-j_{0}(\Delta-1)=n-1-(n-2)(2-1)=1 \text {. }
$$

Additionally,

$$
\Delta j_{0}+n-1-j_{0} \Delta+j_{0}+n-\left(j_{0}+1\right)=2 n-2,
$$

and we conclude $\mathbf{y} \in S_{2 n-2}^{\Delta}$.

Since $n-\Delta \leq n-2$ and

$$
\Delta+2(n-\Delta-1)+n-(n-\Delta)=2 n-2,
$$


we have $\mathbf{z} \in S_{2 n-2}^{\Delta}$.

Seeking for a contradiction assume that

$$
\Delta+2(k-1)=\sum_{i=1}^{k} z_{i}>\sum_{i=1}^{k} x_{i}
$$

for some $k \leq n-\Delta$. Thus, $x_{k}=1$ and we conclude $x_{i}=1$ for every $k \leq i \leq n$, and so,

$$
\sum_{i=1}^{n} x_{i}>\sum_{i=1}^{n} z_{i}=2 n-2
$$

leading to a contradiction. Hence, for every $k \leq n-\Delta$,

$$
\Delta+2(k-1)=\sum_{i=1}^{k} z_{i} \leq \sum_{i=1}^{k} x_{i}
$$

and for every $k>n-\Delta$,

$$
\sum_{i=1}^{k} z_{i}=\sum_{i=1}^{n-\Delta} z_{i}+\sum_{i=n-\Delta+1}^{k} 1 \leq \sum_{i=1}^{n-\Delta} x_{i}+\sum_{i=n-\Delta+1}^{k} x_{i}=\sum_{i=1}^{k} x_{i}
$$

Hence, $\mathbf{z} \prec \mathbf{x}$.

For every $k \leq j_{0}$ it is trivial to check that

$$
\sum_{i=1}^{k} x_{i} \leq \sum_{i=1}^{k} \Delta=\sum_{i=1}^{k} y_{i}
$$

Additionally, since for every $k>j_{0}, x_{k} \geq y_{k}=1$, it is readily seen that

$$
\begin{aligned}
\sum_{i=k+1}^{n} x_{i} & \geq \sum_{i=k+1}^{n} 1=\sum_{i=k+1}^{n} y_{i} \\
\sum_{i=1}^{k} x_{i} & =2 n-2-\sum_{i=k+1}^{n} x_{i} \leq 2 n-2-\sum_{i=k+1}^{n} y_{i}=\sum_{i=1}^{k} y_{i} .
\end{aligned}
$$

Thus, $\mathbf{x} \prec \mathbf{y}$.

Lemma 2 has the following consequences.

Theorem 9. If $T$ is a tree with $n \geq 3$ vertices and maximum degree $\Delta, j_{0}=\left\lfloor\frac{n-2}{\Delta-1}\right\rfloor$ and $f:[1, \infty) \rightarrow \mathbb{R}$ is a convex function, then

$$
f(\Delta)+(n-\Delta-1) f(2)+\Delta f(1) \leq I_{f}(T) \leq j_{0} f(\Delta)+f\left(n-1-j_{0}(\Delta-1)\right)+\left(n-j_{0}-1\right) f(1) .
$$

Moreover, the lower bound is attained if and only if $T$ has the degree sequence $\mathbf{z}$ in Lemma 2, and the upper bound is attained if and only if $T$ has the degree sequence $\mathbf{y}$ in Lemma 2.

Theorem 10. If $T$ is a tree with $n \geq 3$ vertices and maximum degree $\Delta, j_{0}=\left\lfloor\frac{n-2}{\Delta-1}\right\rfloor$ and $f:[1, \infty) \rightarrow \mathbb{R}$ is a concave function, then

$$
j_{0} f(\Delta)+f\left(n-1-j_{0}(\Delta-1)\right)+\left(n-j_{0}-1\right) f(1) \leq I_{f}(T) \leq f(\Delta)+(n-\Delta-1) f(2)+\Delta f(1) .
$$

Moreover, the lower bound is attained if and only if $T$ has the degree sequence $\mathbf{y}$ in Lemma 2, and the upper bound is attained if and only if $T$ has the degree sequence $\mathbf{z}$ in Lemma 2. 
Theorem 11. If $T$ is a tree with $n \geq 3$ vertices and maximum degree $\Delta, j_{0}=\left\lfloor\frac{n-2}{\Delta-1}\right\rfloor$ and $f:[1, \infty) \rightarrow \mathbb{R}^{+}$is a function such that $\log f$ is convex, then

$$
f(\Delta) f(2)^{n-\Delta-1} f(1)^{\Delta} \leq I I_{f}(T) \leq f(\Delta)^{j_{0}} f\left(n-1-j_{0}(\Delta-1)\right) f(1)^{n-j_{0}-1} .
$$

Moreover, the lower bound is attained if and only if $T$ has the degree sequence $\mathbf{z}$ in Lemma 2, and the upper bound is attained if and only if $T$ has the degree sequence $\mathbf{y}$ in Lemma 2.

Theorem 12. If $T$ is a tree with $n \geq 3$ vertices and maximum degree $\Delta, j_{0}=\left\lfloor\frac{n-2}{\Delta-1}\right\rfloor$ and $f:[1, \infty) \rightarrow \mathbb{R}^{+}$is a function such that $\log f$ is concave, then

$$
f(\Delta)^{j_{0}} f\left(n-1-j_{0}(\Delta-1)\right) f(1)^{n-j_{0}-1} \leq I I_{f}(T) \leq f(\Delta) f(2)^{n-\Delta-1} f(1)^{\Delta} .
$$

Moreover, the lower bound is attained if and only if $T$ has the degree sequence $\mathbf{y}$ in Lemma 2, and the upper bound is attained if and only if $T$ has the degree sequence $\mathbf{z}$ in Lemma 2.

Hence, the following results hold.

Theorem 13. If $T$ is a tree with $n \geq 3$ vertices and maximum degree $\Delta, j_{0}=\left\lfloor\frac{n-2}{\Delta-1}\right\rfloor$ and $\alpha \in(-\infty, 0) \cup(1, \infty)$, then

$$
\Delta^{\alpha}+2^{\alpha}(n-\Delta-1)+\Delta \leq M_{1}^{\alpha}(T) \leq \Delta^{\alpha} j_{0}+\left(n-1-j_{0}(\Delta-1)\right)^{\alpha}+n-j_{0}-1 .
$$

Moreover, the lower bound is attained if and only if $T$ has the degree sequence $\mathbf{z}$ in Lemma 2, and the upper bound is attained if and only if $T$ has the degree sequence $\mathbf{y}$ in Lemma 2.

Theorem 14. If $T$ is a tree with $n \geq 3$ vertices and maximum degree $\Delta, j_{0}=\left\lfloor\frac{n-2}{\Delta-1}\right\rfloor$ and $\alpha \in(0,1)$, then

$$
\Delta^{\alpha} j_{0}+\left(n-1-j_{0}(\Delta-1)\right)^{\alpha}+n-j_{0}-1 \leq M_{1}^{\alpha}(T) \leq \Delta^{\alpha}+2^{\alpha}(n-\Delta-1)+\Delta .
$$

Moreover, the lower bound is attained if and only if $T$ has the degree sequence $\mathbf{y}$ in Lemma 2, and the upper bound is attained if and only if $T$ has the degree sequence $\mathbf{z}$ in Lemma 2.

Theorem 15. If $T$ is a tree with $n \geq 3$ vertices and maximum degree $\Delta$, and $j_{0}=\left\lfloor\frac{n-2}{\Delta-1}\right\rfloor$, then

$$
\Delta^{\Delta} 4^{n-\Delta-1} \leq N K^{*}(T) \leq \Delta^{j_{0} \Delta}\left(n-1-j_{0}(\Delta-1)\right)^{n-1-j_{0}(\Delta-1)} .
$$

Moreover, the lower bound is attained if and only if $T$ has the degree sequence $\mathbf{z}$ in Lemma 2, and the upper bound is attained if and only if $T$ has the degree sequence $\mathbf{y}$ in Lemma 2.

Theorem 16. If $T$ is a tree with $n \geq 3$ vertices and maximum degree $\Delta$, and $j_{0}=\left\lfloor\frac{n-2}{\Delta-1}\right\rfloor$, then

$$
\Delta^{j_{0}}\left(n-1-j_{0}(\Delta-1)\right) \leq N K(T) \leq \Delta 2^{n-\Delta-1} .
$$

Moreover, the lower bound is attained if and only if Thas the degree sequence $\mathbf{y}$ in Lemma 2, and the upper bound is attained if and only if $T$ has the degree sequence $\mathbf{z}$ in Lemma 2.

Theorem 13 has the following consequence. 
Corollary 3. If $T$ is a tree with $n \geq 3$ vertices and maximum degree $\Delta$, and $j_{0}=\left\lfloor\frac{n-2}{\Delta-1}\right\rfloor$, then the following inequalities hold:

$$
\begin{aligned}
\Delta^{2}+4(n-\Delta-1)+\Delta & \leq M_{1}(T) \leq \Delta^{2} j_{0}+\left(n-1-j_{0}(\Delta-1)\right)^{2}+n-j_{0}-1, \\
\Delta^{3}+8(n-\Delta-1)+\Delta & \leq F(T) \leq \Delta^{3} j_{0}+\left(n-1-j_{0}(\Delta-1)\right)^{3}+n-j_{0}-1, \\
\Delta^{-1}+2^{-1}(n-\Delta-1)+\Delta & \leq I D(T) \leq \Delta^{-1} j_{0}+\left(n-1-j_{0}(\Delta-1)\right)^{-1}+n-j_{0}-1 .
\end{aligned}
$$

Moreover, each lower bound is attained if and only if $T$ has the degree sequence $\mathbf{z}$ in Lemma 2, and each upper bound is attained if and only if $T$ has the degree sequence $\mathbf{y}$ in Lemma 2.

\section{Wiener Index and Its Generalizations}

The Wiener index of a graph $G$ was defined in [1] as

$$
W(G)=\sum_{\{u, v\} \subseteq V(G)} d(u, v),
$$

where $\{u, v\}$ goes over every pair of distinct vertices in $G$.

In [7], Randić considered a sophisticated version for trees of the Wiener index. Later, this new index has been called the hyper-Wiener index. The generalization to graphs of this index appeared in [6] as

$$
W W(G)=\frac{1}{2} \sum_{\{u, v\} \subseteq V(G)} d(u, v)+\frac{1}{2} \sum_{\{u, v\} \subseteq V(G)} d(u, v)^{2} .
$$

For further results on the hyper-Wiener index, see [2-4]. In particular, $W W(G)$ has shown good correlations with several properties (see, for example, [3] and the references therein).

We are considering the following generalization of the Wiener index.

$$
W^{\lambda}(G)=\sum_{\{u, v\} \subseteq V(G)} d(u, v)^{\lambda},
$$

with $\lambda \in \mathbb{R}$. Clearly, if $\lambda=1$, then $W^{\lambda}$ is just the Wiener index. Additionally, if $\lambda=-1$, it is the reciprocal Wiener index, and if $\lambda=-2$, it is the Harary index. Moreover, the hyper-Wiener index satisfies that $W W=\left(W^{1}+W^{2}\right) / 2$, and therefore it is a simple combination of $W^{1}$ and $W^{2}$. In [8] there appears a topological index which can be defined as $\left(2 W^{1}+3 W^{2}+W^{3}\right) / 6$-that is, a combination of $W^{1}, W^{2}$ and $W^{3}$. More relations of this type can be found in [5].

The following alternatives for the $q$-Wiener index (with $q>0$ and $q \neq 1$ ) appear in [9].

$$
\begin{aligned}
& W_{1}(G, q)=\sum_{\{u, v\} \subseteq V(G)}[d(u, v)]_{q}, \\
& W_{2}(G, q)=\sum_{\{u, v\} \subseteq V(G)}[d(u, v)]_{q} q^{L-d(u, v),} \\
& W_{3}(G, q)=\sum_{\{u, v\} \subseteq V(G)}[d(u, v)]_{q} q^{d(u, v)},
\end{aligned}
$$

where $L$ denotes the diameter of $G$ and

$$
[k]_{q}=\frac{1-q^{k}}{1-q}=1+q+q^{2}+\cdots+q^{k-1} .
$$

Since $\lim _{q \rightarrow 1}[k]_{q}=k$, we have

$$
\lim _{q \rightarrow 1} W_{1}(G, q)=\lim _{q \rightarrow 1} W_{2}(G, q)=\lim _{q \rightarrow 1} W_{3}(G, q)=W(G) .
$$


Given any function $h: \mathbb{Z}^{+} \rightarrow \mathbb{R}$, we define the $h$-Wiener index of $G$ as

$$
W_{h}(G)=\sum_{\{u, v\} \subseteq V(G)} h(d(u, v)),
$$

Through this generalized definition, all these indices and many more can be studied simultaneously.

Notice that if $S_{n}$ is the star graph with $n$ vertices, then

$$
W_{h}\left(S_{n}\right)=(n-1) h(1)+\frac{1}{2}(n-1)(n-2) h(2) .
$$

If $P_{n}$ is the path graph with $n$ vertices, then

$$
W_{h}\left(P_{n}\right)=\sum_{1 \leq i<j \leq n} h(j-i)=\sum_{k=1}^{n-1}(n-k) h(k) .
$$

Let us recall the following definitions from [45]. A major vertex of $G$ is a vertex with degree at least three. A vertex $v$ with degree one is called a terminal vertex of a major vertex $w$ of $G$ if $d_{G}(v, w)<d_{G}(v, u)$ for any other major vertex $u$ in $G$. The number of terminal vertices of a major vertex $w$ is called the terminal degree of $w$. Let $\mathcal{M}(G)$ be the set of major vertices of $G$ with terminal degree greater than one.

Lemma 3. If $T$ is a tree, then $\mathcal{M}(T)=\varnothing$ if and only if $T$ is a path graph.

Proof. The if part is obvious. Suppose $T$ is a tree different from a path graph. Then, $T$ contains a major vertex $v_{1}$. If $v_{1} \notin \mathcal{M}(G)$, then at least two of the components of $T \backslash\left\{v_{1}\right\}$ must contain a major vertex. Let $v_{2}$ be a major vertex in the component $C_{1} \in T \backslash\left\{v_{1}\right\}$. Now, if $v_{2} \notin \mathcal{M}(G)$ then at least two of the components of $T \backslash\left\{v_{2}\right\}$ must contain a major vertex, and at one of them, $C_{2}$ is contained in $C_{1}$. Let $v_{3}$ be a major vertex in $C_{2}$. Then, $v_{3} \neq v_{1}, v_{2}$ and the argument can be repeated. For every $i$ either $v_{i} \in \mathcal{M}(G)$ or there is a connected component $C_{i} \in T \backslash\left\{v_{i}\right\}$ with $C_{i} \subset C_{i-1}$ and a major vertex $v_{i+1} \in C_{i}$ different from $\left\{v_{1}, \ldots, v_{i}\right\}$. Since $T$ is finite, there is some $n$ such that $v_{n} \in \mathcal{M}(G)$.

Given $w \in \mathcal{M}(G)$ and a terminal vertex $u_{j}$ of $w$, let us denote by $\left[u_{j}, w\right]$ the shortest path joining $u_{j}$ and $w$; note that $d\left(u_{j}, w\right)$ is the length of the path $\left[u_{j}, w\right]$.

Theorem 17. Let $T$ be a tree with $n$ vertices.

(1) If $h$ is an increasing function, then

$$
(n-1) h(1)+\frac{1}{2}(n-1)(n-2) h(2) \leq W_{h}(T) \leq \sum_{k=1}^{n-1}(n-k) h(k),
$$

the lower bound is attained if and only if $T$ is the star graph and the upper bound is attained if and only if $T$ is the path graph.

(2) If $h$ is a decreasing function, then

$$
\sum_{k=1}^{n-1}(n-k) h(k) \leq W_{h}(T) \leq(n-1) h(1)+\frac{1}{2}(n-1)(n-2) h(2)
$$

the lower bound is attained if and only if $T$ is the path graph and the upper bound is attained if and only if $T$ is the star graph.

Proof. Assume that $h$ is an increasing function. 
Since $T$ is a tree with $n$ vertices, there are $n-1$ edges, and therefore, $n-1$ pairs of adjacent vertices and $\left(\begin{array}{c}n \\ 2\end{array}\right)-(n-1)=\frac{1}{2}(n-1)(n-2)$ pairs of vertices at distance at least 2 . Thus, $W_{h}(T) \geq$ $(n-1) h(1)+\frac{1}{2}(n-1)(n-2) h(2)$. This bound is attained if and only if $T$ does not contain a path of length 3; that is, if and only if $T$ is the star graph.

Assume that $T$ is a tree with $n$ vertices such that $W_{h}(T)$ is maximal and suppose that $T$ contains a vertex with degree at least 3 . Then, by Lemma 3, there is a vertex $w \in \mathcal{M}(T)$ and two terminal vertices of $w, u_{1}, u_{2}$ with $d\left(w, u_{1}\right)=k$. Consider new vertices $\left\{v_{i}: 1 \leq i \leq k\right\}$ and let

$$
T_{1}:=\left(T \backslash\left[w, u_{1}\right]\right) \cup u_{2} v_{1} \cup v_{1} v_{2} \cup \cdots \cup v_{k-1} v_{k} .
$$

Notice that the subgraph $\left[u_{1}, u_{2}\right]$ in $T$ and the subgraph $\left[w, v_{k}\right]$ in $T_{1}$ are both paths with the same length. Hence, the restriction of $W_{h}$ in these subgraphs is equal and it is urgent to check that $W_{h}\left(T_{1}\right)>W_{h}(T)$ (since $h$ is an increasing function), leading to a contradiction. Therefore, the maximum degree of $T$ is less than 3 , and so, $T$ is the path graph.

If $h$ is a decreasing function, then a similar argument gives the result.

Theorem 18. Let $G$ be a graph with $n$ vertices.

(1) If $h$ is an increasing function, then

$$
\frac{1}{2} n(n-1) h(1) \leq W_{h}(G) \leq \sum_{k=1}^{n-1}(n-k) h(k),
$$

the equality is attained in the upper bound if and only if $G$ is the path graph.

(2) If $h$ is a decreasing function, then

$$
\sum_{k=1}^{n-1}(n-k) h(k) \leq W_{h}(G) \leq \frac{1}{2} n(n-1) h(1),
$$

the equality is attained in the upper bound if and only if $G$ is the complete graph.

Proof. Assume that $h$ is an increasing function.

Since $G$ is a graph with $n$ vertices, there are $\left(\begin{array}{l}n \\ 2\end{array}\right)$ pairs of vertices at distance at least 1 . Since $h$ is an increasing function, we have $W_{h}(T) \geq\left(\begin{array}{l}n \\ 2\end{array}\right) h(1)$, and the equality is attained if and only if every pair of vertices is at distance 1 ; i.e., $G$ is the complete graph.

Let us prove now the upper bound. If $G$ is a tree, then Theorem 17 gives the inequality. Assume now that $G$ is not a tree. Thus, there exists an edge $u v \in E(G)$ such that the graph $G \backslash u v$ (defined by $V(G \backslash u v)=V(G)$ and $E(G \backslash u v)=E(G) \backslash\{u v\})$ is connected. Since $d_{G \backslash u v}\left(w_{1}, w_{2}\right) \geq d_{G}\left(w_{1}, w_{2}\right)$ for every $w_{1}, w_{2} \in V(G)$ and $d_{G \backslash u v}(u, v)>1=d_{G}(u, v)$, we conclude $W_{h}(G \backslash u v)>W_{h}(G)$. By applying this argument a finite number of times, we obtain a tree $T$ with $W_{h}(T)>W_{h}(G)$, and so Theorem 17 gives the result.

If $h$ is a decreasing function, then a similar argument gives the result.

Given any $2 \leq \Delta \leq n-1$, let $T_{n}^{\Delta}$ be the tree with $n$ vertices obtained from the star graph $S_{\Delta+1}$ and the path graph $P_{n-\Delta}$, where a vertex with degree 1 in each graph is identified. Note that $T_{n}^{2}=P_{n}$ and $T_{n}^{n-1}=S_{n}$.

In $T_{n}^{\Delta}$ there are $\Delta-1$ vertices, $y_{1}, \ldots, y_{\Delta-1}$, with degree 1 adjacent to the vertex with degree $\Delta$, and for these vertices, the distance between any pair is 2 . Then, there are $\left(\begin{array}{c}\Delta-1 \\ 2\end{array}\right)$ pairs at distance 2 . $T_{n}^{\Delta} \backslash\left\{y_{1}, \ldots, y_{\Delta-1}\right\}$ is a path with $n-\Delta+1$ vertices. Finally, for each $y_{i}$, there is one vertex in $T_{n}^{\Delta} \backslash\left\{y_{1}, \ldots, y_{\Delta-1}\right\}$ at distance $k$ for every $1 \leq k \leq n-\Delta+1$. Thus, 


$$
\begin{aligned}
W_{h}\left(T_{n}^{\Delta}\right) & =\left(\begin{array}{c}
\Delta-1 \\
2
\end{array}\right) h(2)+(\Delta-1) \sum_{k=1}^{n-\Delta+1} h(k)+W_{h}\left(P_{n-\Delta+1}\right) \\
& =\frac{1}{2}(\Delta-1)(\Delta-2) h(2)+(\Delta-1) \sum_{k=1}^{n-\Delta+1} h(k)+\sum_{k=1}^{n-\Delta}(n-\Delta+1-k) h(k) \\
& =\frac{1}{2}(\Delta-1)(\Delta-2) h(2)+\sum_{k=1}^{n-\Delta+1}(n-k) h(k) .
\end{aligned}
$$

Theorem 19. Let $T$ is a tree with $n$ vertices and maximum degree $\Delta \geq 2$.

(1) If $h$ is an increasing function, then

$$
W_{h}(T) \leq \frac{1}{2}(\Delta-1)(\Delta-2) h(2)+\sum_{k=1}^{n-\Delta+1}(n-k) h(k),
$$

and the equality is attained if and only if $T=T_{n}^{\Delta}$.

(2) If $h$ is a decreasing function, then

$$
W_{h}(T) \geq \frac{1}{2}(\Delta-1)(\Delta-2) h(2)+\sum_{k=1}^{n-\Delta+1}(n-k) h(k),
$$

and the equality is attained if and only if $T=T_{n}^{\Delta}$.

Proof. Assume that $h$ is an increasing function.

If $\Delta=2$ or $\Delta=n-1$, then $T$ is the path graph or the star graph, respectively, and the inequality is, in fact, an equality. Hence, we can assume that $2<\Delta<n-1$.

Let us consider a maximal $T$ for $W_{h}$. Since $\Delta>2$, Lemma 3 gives $k=|\mathcal{M}(T)| \geq 1$. If $k \geq 2$, the same argument from the proof of the upper bound in Theorem 17 shows that there exists a tree $T^{\prime}$ such that $W_{h}\left(T^{\prime}\right)>W_{h}(T)$. Thus, $|\mathcal{M}(T)|=1$, and so, if $w$ is the vertex with $\mathcal{M}(T)=\{w\}$, then $T$ is the union of $\Delta$ paths $\left[w, w_{1}\right], \ldots,\left[w, w_{\Delta}\right]$. Since $\Delta<n-1$, there exists $w_{i}$ with $d\left(w_{i}, w\right)>1$. Seeking for a contradiction assume that $d\left(w_{j}, w\right)>1$ for some $j \neq i(1 \leq j \leq \Delta)$ with $d\left(w, w_{j}\right) \leq d\left(w, w_{i}\right)$ (relabeling if necessary). Let $z$ be a new vertex and consider the graph $T^{\prime}$ obtained from $T \cup\left\{w_{i} z\right\}$ by removing the edge incident to $w_{j}$. Hence, $T^{\prime}$ is also a tree with $n$ vertices and maximum degree $\Delta$, and $W_{h}\left(T^{\prime}\right)>W_{h}(T)$. Thus, $d\left(w_{j}, w\right)=1$ for every $j \neq i$, and so, $T=T_{n}^{\Delta}$.

If $h$ is a decreasing function, then a similar argument gives the result.

Theorem 20. Let $G$ be a graph with $n$ vertices and maximum degree $\Delta \geq 2$.

(1) If $h$ is an increasing function, then

$$
W_{h}(G) \leq \frac{1}{2}(\Delta-1)(\Delta-2) h(2)+\sum_{k=1}^{n-\Delta+1}(n-k) h(k),
$$

and the equality is attained if and only if $G=T_{n}^{\Delta}$.

(2) If $h$ is a decreasing function, then

$$
W_{h}(G) \geq \frac{1}{2}(\Delta-1)(\Delta-2) h(2)+\sum_{k=1}^{n-\Delta+1}(n-k) h(k),
$$

and the equality is attained if and only if $G=T_{n}^{\Delta}$.

Proof. Assume that $h$ is an increasing function. 
If $G$ is a tree, then Theorem 19 gives the inequality. Assume now that $G$ is not a tree. Fix a vertex $w \in V(G)$ with degree $\Delta$. Thus, there exists an edge $u v \in E(G)$ such that $w \notin\{u, v\}$ and $G \backslash u v$ is connected. Note that the graph $G \backslash u v$ has $n$ vertices and maximum degree $\Delta$, since the degree of $w$ in $G \backslash u v$ is $\Delta$. Since $d_{G \backslash u v}\left(w_{1}, w_{2}\right) \geq d_{G}\left(w_{1}, w_{2}\right)$ for every $w_{1}, w_{2} \in V(G)$ and $d_{G \backslash u v}(u, v)>1=d_{G}(u, v)$, we conclude $W_{h}(G \backslash u v)>W_{h}(G)$. By applying this argument a finite number of times, we obtain a tree $T$ with $n$ vertices, maximum degree $\Delta$ and $W_{h}(T)>W_{h}(G)$. Thus, Theorem 19 gives the result.

If $h$ is a decreasing function, then a similar argument gives the result.

\section{Conclusions}

A typical problem in the study of topological indices is finding good bounds for some indices, when some parameters of the graph are known. In this paper we use a unified approach in order to prove new inequalities when the graph is a tree for a wide family of topological indices. Additionally, we characterize those trees which are extremal for them.

First of all we obtain, by using Schur-convexity, the extremal trees with $n$ vertices for a large family of topological indices (see Theorems 1-4); these results are applied for the first variable Zagreb, the Narumi-Katayama and the modified Narumi-Katayama indices in Theorems 5-8. The Schur-convexity also allows us to find the extremal trees with $n$ vertices and maximum degree $\Delta$ for the previous family of topological indices (see Theorems 9-12); these results are applied for the first variable Zagreb, the Narumi-Katayama and the modified Narumi-Katayama indices in Theorems 13-16.

Additionally, we obtain similar results on extremal trees with $n$ vertices for a large class of topological indices, including the Wiener index and many generalizations of this index (see Theorems 17 and 18); Theorems 19 and 20 provide similar results for trees with $n$ vertices and maximum degree $\Delta$.

Finally, we want to remark on a new direction for future research. We are studying how to extend these results for indices defined by

$$
\sum_{u v \in E(G)} F\left(d_{u}, d_{v}\right)
$$

for an appropriate function $F$. These indices include, for instance, the case of the second variable Zagreb index.

Author Contributions: Both authors contributed equally with the ideas and writing of this paper. Both authors have read and agreed to the published version of the manuscript

Funding: The first author was partially supported by a grant from Ministerio de Ciencia, Innovación y Universidades (PGC2018-098321-B-I00), Spain; the second author was partially supported by two grants from Ministerio de Economía y Competitividad, Agencia Estatal de Investigación (AEI) and Fondo Europeo de Desarrollo Regional (FEDER) (MTM2016-78227-C2-1-P and MTM2017-90584-REDT), Spain.

Acknowledgments: We would like to thank the referees for their careful reading and for some useful suggestions which have improved the presentation of the manuscript.

Conflicts of Interest: The authors declare no conflict of interest.

\section{References}

1. Wiener, H. Structural determination of paraffin boiling points. J. Am. Chem. Soc. 1947, 69, 17-20. [CrossRef]

2. Cash, G.G. Relationship between the Hosoya polynomial and the hyper-Wiener index. Appl. Math. Lett. 2002, 15, 893-895. [CrossRef]

3. Gutman, I. Relation between hyper-Wiener and Wiener index. Chem. Phys. Lett. 2002, 364, 352-356. [CrossRef]

4. Klavzar, S.; Zigert, P.; Gutman, I. An algorithm for the calculation of the hyper-Wiener index of benzenoid hydrocarbons. Comput. Chem. 2000, 24, 229-233. [CrossRef]

5. Klein, D.J.; Gutman, I. Wiener-Number-Related Sequences. J. Chem. Inf. Comput. Sci. 1999, 39, $534-536$. [CrossRef] 
6. Klein, D.J.; Lukovits, I.; Gutman, I. On the definition of the hyper-Wiener index for cycle-containing structures. J. Chem. Inf. Comput. Sci. 1995, 35, 50-52. [CrossRef]

7. Randić, M. Novel molecular descriptor for structure-property studies. Chem. Phys. Lett. 1993, 211, 478-483. [CrossRef]

8. Tratch, S.S.; Stankevich, M.I.; Zefirov, N.S. Combinatorial Models and Algorithms in Chemistry. The Expanded Wiener Numbers-A Novel Topological Index. J. Comput. Chem. 1990, 11, 899-908. [CrossRef]

9. Zhang, Y.S.; Gutman, I.; Liu, J.G.; Mu, Z.C. q-Analog of Wiener index. MATCH Commun. Math. Comput. Chem. 2012, 67, 347-356.

10. Randić, M. On characterization of molecular branching. J. Am. Chem. Soc. 1975, 97, 6609-6615. [CrossRef]

11. Gutman, I.; Furtula, B. (Eds.) Recent Results in the Theory of Randic Index; Univ. Kragujevac: Kragujevac, Serbia, 2008.

12. Li, X.; Gutman, I. Mathematical Aspects of Randić Type Molecular Structure Descriptors; Univ. Kragujevac: Kragujevac, Serbia, 2006.

13. Li, X.; Shi, Y. A survey on the Randić index. MATCH Commun. Math. Comput. Chem. 2008, 59, 127-156.

14. Rodríguez-Velázquez, J.A.; Sigarreta, J.M. On the Randić index and condicional parameters of a graph. MATCH Commun. Math. Comput. Chem. 2005, 54, 403-416.

15. Rodríguez-Velázquez, J.A.; Tomás-Andreu, J. On the Randić index of polymeric networks modelled by generalized Sierpinski graphs. MATCH Commun. Math. Comput. Chem. 2015, 74, 145-160.

16. Gutman, I.; Trinajstić, N. Graph theory and molecular orbitals. Total $\pi$-electron energy of alternant hydrocarbons. Chem. Phys. Lett. 1972, 17, 535-538. [CrossRef]

17. Miličević, A.; Nikolić, S. On variable Zagreb indices. Croat. Chem. Acta 2004, 77, 97-101.

18. Gutman, I.; Rodríguez, J.M.; Sigarreta, J.M. Linear and non-linear inequalities on the inverse sum indeg index. Discr. Appl. Math. 2019, 258, 123-134. [CrossRef]

19. Martínez-Pérez, A.; Rodríguez, J.M.; Sigarreta, J.M. A new approximation to the geometric-arithmetic index. J. Math. Chem. 2018, 56, 1865-1883. [CrossRef]

20. Rodríguez, J.M.; Rodríguez-Velázquez, J.A.; Sigarreta, J.M. New Inequalities Involving the GeometricArithmetic Index. MATCH Commun. Math. Comput. Chem. 2017, 78, 361-374.

21. Rodríguez, J.M.; Sánchez, J.L.; Sigarreta, J.M. On the first general Zagreb index. J. Math. Chem. 2018, 56, 1849-1864. [CrossRef]

22. Rodríguez, J.M.; Sánchez, J.L.; Sigarreta, J.M. Inequalities on the inverse degree index. J. Math. Chem. 2019, 57, 1524-1542. [CrossRef]

23. Rodríguez, J.M.; Sigarreta, J.M. New Results on the Harmonic Index and Its Generalizations. MATCH Commun. Math. Comput. Chem. 2017, 78, 387-404.

24. Sigarreta, J.M. Bounds for the geometric-arithmetic index of a graph. Miskolc Math. Notes 2015, 16, 1199-1212. [CrossRef]

25. Fajtlowicz, S. On conjectures of Graffiti-II. Congr. Numer. 1987, 60, 187-197.

26. Nikolić, S.; Kovačević, G.; Miličević, A.; Trinajstić, N. The Zagreb Indices 30 years after. Croat. Chem. Acta 2003, 76, 113-124.

27. Randić, M. Novel graph theoretical approach to heteroatoms in QSAR. Chemom. Intell. Lab. Syst. 1991, 10, 213-227. [CrossRef]

28. Randić, M. On computation of optimal parameters for multivariate analysis of structure-property relationship. J. Chem. Inf. Comput. Sci. 1991, 31, 970-980. [CrossRef]

29. Randić, M.; Plavšić, D.; Lerš, N. Variable connectivity index for cycle-containing structures. J. Chem. Inf. Comput. Sci. 2001, 41, 657-662. [CrossRef]

30. Gutman, I.; Tosovic, J. Testing the quality of molecular structure descriptors. Vertex-degreebased topological indices. J. Serb. Chem. Soc. 2013, 78, 805-810. [CrossRef]

31. Nikolić, S.; Miličević, A.; Trinajstić, N.; Jurić, A. On Use of the Variable Zagreb ${ }^{v} M_{2}$ Index in QSPR: Boiling Points of Benzenoid Hydrocarbons. Molecules 2004, 9, 1208-1221. [CrossRef]

32. Liu, M.; Liu, B. Some properties of the first general Zagreb index. Australas. J. Combin. 2010, 47, $285-294$.

33. Li, X.; Zhao, H. Trees with the first smallest and largest generalized topological indices. MATCH Commun. Math. Comput. Chem. 2004, 50, 57-62.

34. Singh, M.; Das, K.C.; Gupta, S.; Madan, A.K. Refined variable Zagreb indices: Highly discriminating topological descriptors for QSAR/QSPR. Int. J. Chem. Model. 2014, 6, 403-428. 
35. Andova, V.; Petrusevski, M. Variable Zagreb Indices and Karamata's Inequality. MATCH Commun. Math. Comput. Chem. 2011, 65, 685-690.

36. Zhang, S.; Wang, W.; Cheng, T.C.E. Bicyclic graphs with the first three smallest and largest values of the first general Zagreb index. MATCH Commun. Math. Comput. Chem. 2006, 55, 579-592.

37. Zhang, H.; Zhang, S. Unicyclic graphs with the first three smallest and largest values of the first general Zagreb index. MATCH Commun. Math. Comput. Chem. 2006, 55, 427-438.

38. Bollobás, B.; Erdös, P. Graphs of extremal weights. Ars Comb. 1998, 50, 225-233. [CrossRef]

39. Bollobás, B.; Erdös, P.A. Sarkar, Extremal graphs for weights. Discr. Math. 1999, 200, 5-19. [CrossRef]

40. Eliasi, M.; Ghalavand, A. Extremal Trees with Respect to Some Versions of Zagreb Indices Via Majorization. Iran. J. Math. Chem. 2017, 8, 391-401.

41. Gutman, I.; Das, K.C. The first Zagreb index 30 years after. MATCH Commun. Math. Comput. Chem. 2004, 50, 83-92.

42. Narumi, H.; Katayama, M. Simple topological index. A newly devised index characterizing the topological nature of structural isomers of saturated hydrocarbons. Mem. Fac. Eng. Hokkaido Univ. 1984, 16, 209-214.

43. Ghorbani, M.; Songhori, M.; Gutman, I. Modified Narumi-Katayama index. Kragujevac J. Sci. 2012, 34, 57-64.

44. Gutman, I.; Ghorbani, M. Some properties of the Narumi-Katayama index. Appl. Math. Lett. 2012, 25, 1435-1438. [CrossRef]

45. Estrada-Moreno, A.; Rodríguez-Velázquez, J.A.; Yero, I.G. The k-metric dimension of a graph. Appl. Math. Inf. Sci. 2015, 9, 2829-2840.

(C) 2020 by the authors. Licensee MDPI, Basel, Switzerland. This article is an open access article distributed under the terms and conditions of the Creative Commons Attribution (CC BY) license (http://creativecommons.org/licenses/by/4.0/). 